\title{
Proton Single Particle Energy Shifts due to Coulomb Correlations
}

\author{
Aurel Bulgac ${ }^{1}$ and Vasily R. Shaginyan ${ }^{1,2}$ \\ 1 Department of Physics, University of Washington, Seattle, WA 98195, USA \\ ${ }^{2}$ Petersburg Nuclear Physics Institute, 188350 Gatchina, RUSSIA
}

\begin{abstract}
A theoretically consistent approach to the calculation of the Coulomb correlation corrections to the single-particle energies is presented. New contributions to the single-particle energies previously overlooked in the literature are now identified and taken into account. We show that the interplay between the Coulomb interaction and the strong interaction, which is enhanced in the nuclear surface, leads to an upward shift of the proton single-particle levels. This shift affects the position of the calculated proton drip line, a shift towards decreasing $Z$. We describe briefly a similar mechanism which is at work for neutron levels. The same mechanism is responsible for significant corrections to the mass difference of the mirror nuclei (Nolen-Schiffer anomaly) and to the effective proton mass.
\end{abstract}

PACS: 21.10.Sf Coulomb energies - 21.10.Dr Binding energies - 21.10.-k Nuclear energy levels

The main part of the Coulomb energy in nuclei is given by the Hartree contribution and to a reasonable accuracy this can be computed as the energy of a uniformly charged sphere and is thus proportional to $Z^{2} / A^{1 / 3}$. There are a number of corrections, some of them rather subtle, arising from the interplay between the Coulomb and nuclear forces. The Nolen-Schiffer anomaly in the binding energy of mirror nuclei [1] is a case in point. In Ref. [2] we have shown that a specific many-body mechanism (outlined concisely below) leads to an enhancement of the Coulomb energy in the nuclear surface region and in this way one can account for a major part of this anomaly. This effect results in a systematic contribution to the nuclear binding energy, which scales as $\propto Z^{2 / 3}$. We are going to demonstrate that the 
mechanism should be taken into account when calculating a number of nuclear properties. In this Letter we study the role of this new many-body effect on the single-particle proton energy levels, on the location of the proton drip line and on the proton effective mass.

We shall operate within the density functional theory [3 6]. The ground state energy of a nucleus $E$ is given by a sum of two functionals (in the absence of pairing correlations):

$$
E=F_{0}\left[\rho_{\pi}(\mathbf{r}), \rho_{\nu}(\mathbf{r})\right]+F_{\text {Coul }}\left[\rho_{\pi}(\mathbf{r}), \rho_{\nu}(\mathbf{r})\right]
$$

where the symmetric part $F_{0}\left[\rho_{\pi}(\mathbf{r}), \rho_{\nu}(\mathbf{r})\right]=F_{0}\left[\rho_{\nu}(\mathbf{r}), \rho_{\pi}(\mathbf{r})\right]$ is due to the (strong) isospin conserving nuclear forces, while $F_{\text {Coul }}$ is due to the (weak) Coulomb interaction. We shall neglect in our analysis several easy to include terms: the trivial contribution in the kinetic energy, arising from proton-neutron mass difference, the contribution of the Charge Symmetry Breaking (CSB) forces [7]. For the sake of simplicity of the presentation, we shall not display explicitly the spin degrees of freedom and the contribution arising from the spinorbit interaction, even though we have included them in the actual calculations. The proton and neutron densities are defined as usual

$$
\begin{aligned}
& \rho_{\pi}(\mathbf{r})=\sum_{l} n_{\pi l}\left|\phi_{\pi l}(\mathbf{r})\right|^{2} \\
& \rho_{\nu}(\mathbf{r})=\sum_{l} n_{\nu l}\left|\phi_{\nu l}(\mathbf{r})\right|^{2}
\end{aligned}
$$

where $n_{\pi l}, \phi_{\pi l}$ and $n_{\nu l}, \phi_{\nu l}$ are proton and neutron quasiparticle occupation numbers and single-particle wave functions respectively. The well known Skyrme functional, see e.g. Refs. [8.9], can be considered as one possible realization of $F_{0}$. The Coulomb energy functional is given by:

$$
\begin{aligned}
F_{\text {Coul }} & =e^{2} \int d \mathbf{r}_{1} d \mathbf{r}_{2} \frac{\rho_{\pi \pi}\left(\mathbf{r}_{1}, \mathbf{r}_{2}\right)}{\left|\mathbf{r}_{1}-\mathbf{r}_{2}\right|}=F_{\text {Coul }}^{\text {Hartree }}+F_{\text {Coul }}^{\text {Fock }}+F_{\text {Coul }}^{\text {corr }}, \\
F_{\text {Coul }}^{\text {Hartree }} & =\frac{e^{2}}{2} \int d \mathbf{r}_{1} d \mathbf{r}_{2} \frac{\rho_{\pi}\left(\mathbf{r}_{1}\right) \rho_{\pi}\left(\mathbf{r}_{2}\right)}{\left|\mathbf{r}_{1}-\mathbf{r}_{2}\right|} \\
F_{\text {Coul }}^{\text {Fock }} & =-\frac{e^{2}}{2} \int \frac{d \mathbf{r}_{1} d \mathbf{r}_{2} d \omega}{2 \pi} \frac{\chi_{\pi}^{0}\left(\mathbf{r}_{1}, \mathbf{r}_{2}, i \omega\right)+2 \pi \rho_{\pi}\left(\mathbf{r}_{1}\right) \delta\left(\mathbf{r}_{1}-\mathbf{r}_{2}\right) \delta(\omega)}{\left|\mathbf{r}_{1}-\mathbf{r}_{2}\right|} \\
F_{\text {Coul }}^{\text {corr }} & =-\frac{e^{2}}{2} \int \frac{d \mathbf{r}_{1} d \mathbf{r}_{2} d \omega}{2 \pi} \frac{\chi_{\pi \pi}\left(\mathbf{r}_{1}, \mathbf{r}_{2}, i \omega\right)-\chi_{\pi}^{0}\left(\mathbf{r}_{1}, \mathbf{r}_{2}, i \omega\right)}{\left|\mathbf{r}_{1}-\mathbf{r}_{2}\right|}
\end{aligned}
$$


with $\rho_{\pi \pi}\left(\mathbf{r}_{1}, \mathbf{r}_{2}\right)$ being the exact two-proton density distribution function. $\chi_{\pi \pi}\left(\mathbf{r}_{1}, \mathbf{r}_{2}, i \omega\right)$ and $\chi_{\pi}^{0}\left(\mathbf{r}_{1}, \mathbf{r}_{2}, i \omega\right)$ are the full and free proton linear response functions respectively, evaluated at the imaginary frequency $i \omega$. Eqs. (5) and (6) represent the Hartree and Fock contributions to the Coulomb energy. The exchange term is written here in a somewhat unusual way [2], through the linear response function of the noninteracting protons, $\chi_{\pi}^{0}$, since upon integrating along the real axis of the complex $\omega$ plane one has

$$
\int_{0}^{\infty} \frac{d \omega}{\pi} \operatorname{Im} \chi_{\pi}^{0}\left(\mathbf{r}_{1}, \mathbf{r}_{2}, \omega\right)=\left[\rho_{\pi}\left(\mathbf{r}_{1}, \mathbf{r}_{2}\right) \rho_{\pi}\left(\mathbf{r}_{2}, \mathbf{r}_{1}\right)-\delta\left(\mathbf{r}_{1}-\mathbf{r}_{2}\right) \rho_{\pi}\left(\mathbf{r}_{1}\right)\right]
$$

where $\rho_{\pi}\left(\mathbf{r}_{1}, \mathbf{r}_{2}\right)$ is the proton single-particle density matrix [10. In Eqs. (66,7) we have performed a Wick rotation in order to evaluate the integral along the imaginary axis. Upon taking into account the (strong) residual interaction, the linear free response function $\chi_{\pi}^{0}$ should be replaced with the full response function $\chi_{\pi \pi}$ and one thus readily obtains the expression for the Coulomb correlation energy (7). By considering all three contributions (5, 6, (7) we therefore account for all diagrams in first order in $e^{2}$, in the (weak) Coulomb interaction. The (strong) nuclear interaction is treated to all orders. We are going to concentrate on a study of the contribution to the single particle energies and the effective mass due to Eq. (7). In passing we remark that there are no ambiguities with double counting, rearrangement energy, core-polarization correction and so forth, see Refs. [11] for a discussion of this issues, within the density functional formalism.

By expressing $F_{C o u l}^{c o r r}$ through the linear response function we can easily calculate a number of functional derivatives needed below. At the same time one can clearly disentangle the contribution of various modes to this part of the energy density functional as well [4]. In Ref. [2] we have shown that the main contribution to $F_{C o u l}^{c o r r}$ comes from the surface collective isoscalar excitations. In the isoscalar channel the particle-hole residual interaction has a strong density dependence, changing from a relatively weak one inside nuclei to a strong attractive one in the surface region [15]. Because of the attractive character of the isoscalar residual particle-hole interaction, the nuclear surface (where the matter density is low) is very close to instability [2]. Low density homogeneous nuclear matter is manifestly unstable 
and this shows itself in the fact that the linear response function has a pole for imaginary values of $\omega$ [2]. In finite nuclei and semi-infinite nuclear matter this singularity is smoothed out and becomes a prominent peak of the response function at low frequencies in the surface region. This proximity of the nuclear surface to instability is the reason why the contribution of the (weak) Coulomb exchange interaction is strongly enhanced and the correlation Coulomb "correction" to the mass formula has as a result a predominantly surface character, i.e. $F_{C o u l}^{c o r r} \propto Z^{2 / 3}$.

Let us turn now to the calculation of the proton single-particle energy corrections due to the presence of $F_{C o u l}^{\text {corr }}$ in the energy functional. Using Landau's variational equation [16]

$$
\varepsilon_{\pi l}=\frac{\delta E}{\delta n_{\pi l}}
$$

one obtains for the proton single-particle energy shift $\Delta \varepsilon_{\pi l}$ the following expression

$$
\Delta \varepsilon_{\pi l}=-\frac{e^{2}}{2} \int \frac{d \mathbf{r}_{1} d \mathbf{r}_{2} d \omega}{2 \pi\left|\mathbf{r}_{1}-\mathbf{r}_{2}\right|} \frac{\delta\left[\chi_{\pi \pi}\left(\mathbf{r}_{1}, \mathbf{r}_{2}, i \omega\right)-\chi_{\pi}^{0}\left(\mathbf{r}_{1}, \mathbf{r}_{2}, i \omega\right)\right]}{\delta n_{\pi l}} .
$$

The variational derivative $\delta \chi_{\pi}^{0} / \delta n_{\pi l}$ has the simple functional form,

$$
\frac{\delta \chi_{\pi}^{0}\left(\mathbf{r}_{1}, \mathbf{r}_{2}, i \omega\right)}{\delta n_{\pi \lambda_{0}}}=\left[G_{\pi}\left(\mathbf{r}_{1}, \mathbf{r}_{2}, i \omega+\varepsilon_{\pi \lambda_{0}}\right)+(\omega \rightarrow-\omega)\right] \phi_{\pi \lambda_{0}}^{*}\left(\mathbf{r}_{1}\right) \phi_{\pi \lambda_{0}}\left(\mathbf{r}_{2}\right),
$$

with $G_{\pi}\left(\mathbf{r}_{1}, \mathbf{r}_{2}, \omega\right)$ being the single-particle proton propagator in the selfconsistent nuclear potential. The linear response function $\chi_{\pi \pi}$ is obtained by solving the usual matrix functional equation (Landau zero sound or RPA)

$$
\chi_{i j}=\chi_{i}^{0} \delta_{i j}+\sum_{k=\pi, \nu} \chi_{i}^{0} R_{i k} \chi_{k j}
$$

where $R_{i k}$ is the irreducible (strong) particle-hole interaction and $i, j$ and $k$ stand for the isospin variables, $\pi$ and $\nu$ for protons and neutrons respectively. From this equation one derives the following equation for $\delta \chi_{\pi \pi} / \delta n_{\pi}$

$$
\frac{\delta \chi_{i j}}{\delta n_{\pi l}}=\frac{\delta \chi_{i}^{0}}{\delta n_{\pi l}} \delta_{i j}+\sum_{k=\pi, \nu}\left[\frac{\delta \chi_{m}^{0}}{\delta n_{\pi l}} R_{i k} \chi_{k j}+\chi_{i}^{0} \frac{\delta R_{i k}}{\delta n_{\pi l}} \chi_{k j}+\chi_{i}^{0} R_{i k} \frac{\delta \chi_{k j}}{\delta n_{\pi l}}\right] .
$$

The operator solution can be easily obtained and one thus can show that the relevant quantity in the integrand of Eq. (10) has the following structure 


$$
\begin{aligned}
& \frac{\delta \chi}{\delta n_{\pi} l}-\frac{\delta \chi_{0}}{\delta n_{\pi l}}=\frac{1}{1-\chi_{0} R} \frac{\delta \chi^{0}}{\delta n_{\pi l}} \frac{1}{1-R \chi_{0}}-\frac{\delta \chi_{0}}{\delta n_{\pi l}}+\chi \frac{\delta R}{\delta n_{\pi l}} \chi \\
= & \frac{\delta \chi_{0}}{\delta n_{\pi l}} R \chi+\chi R \frac{\delta \chi_{0}}{\delta n_{\pi l}}+\chi R \frac{\delta \chi_{0}}{\delta n_{\pi l}} R \chi+\chi \frac{\delta R}{\delta n_{\pi l}} \chi
\end{aligned}
$$

where for the sake of clarity we have suppressed the isospin, spin and spatial coordinates and the corresponding summations and integrations.
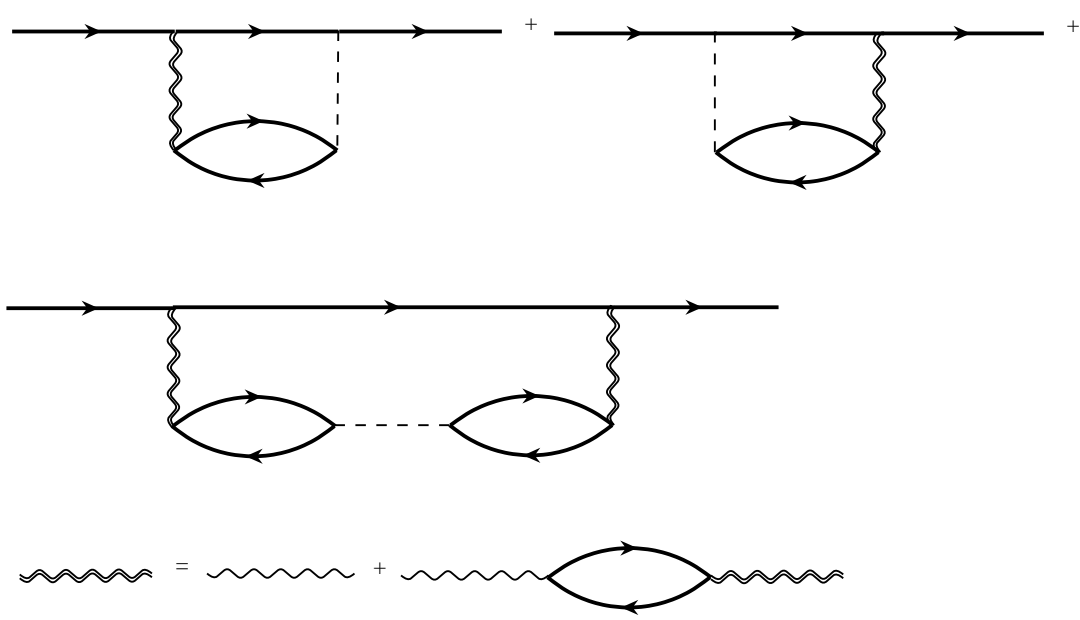

FIG. 1. The diagrammatic representation of corrections to the single-particle nucleon propagator, corresponding to the Coulomb correlation energy discussed in this Letter. The solid oriented lines represent the nucleon propagators, the dashed lines the Coulomb interaction, the thin wavy line the strong nuclear interaction $R_{i k}\left(\mathbf{r}_{1}, \mathbf{r}_{2}\right)$ and the double wavy lines stand for the "screened" strong nuclear interaction, obtained by solving the diagrammatic equation shown in the third row.

In Fig. 1 we show the diagrammatic representation of the corrections to the singleparticle nucleon propagator, corresponding to the addition of the Coulomb correlation energy to the nuclear density functional given by Eq. (7). Strictly speaking, we have omitted in this diagrammatic representation the contribution arising from the last term in Eq. (14), which depends on the functional derivative of the irreducible particle-hole interaction $\delta R / \delta n_{\pi l}$. We shall discuss in more detail the relevance of such a correction in a future publication. To the best of our knowledge this correction was not explicitly discussed in literature in this context. A superficial analysis would suggest that this type of correction is perhaps small. The two diagrams shown in the upper row were considered before by other authors 
[11], however the third diagram, shown in the middle row, was, surprisingly, overlooked. All three diagrams are of the same order in the electromagnetic coupling constant $e^{2}$. Only when considering all three diagrams does the correction to the single-particle energy satisfy the natural Landau's variational equation (9). Besides being theoretically consistent, the present approach has a new physical feature as well: there are corrections to the singleparticle neutron properties too. While for protons all three diagrams have to be considered, for neutrons there is a Coulomb correlation correction to the single-particle energies arising from the third diagram alone.

We do not consider in this Letter the so called AKW diagrams [12], which are automatically taken into account in selfconsistent calculations of nuclear masses [9]. Auerbach [13] has shown that the corrections corresponding to these diagrams are too small to account for the Nolen-Shiffer anomaly.

In Ref. [2] we have used a simple separable model for the residual interaction $R_{i k}$

$$
R_{i k}\left(\mathbf{r}_{1}, \mathbf{r}_{2}\right)=\lambda \frac{d V_{i}\left(r_{1}\right)}{d r} \frac{d V_{k}\left(r_{2}\right)}{d r} \delta\left(\Omega_{1}-\Omega_{2}\right),
$$

where $V_{i}(r)$ is the proton/neutron single-particle potential in a spherical nucleus and $\delta\left(\Omega_{1}-\right.$ $\left.\Omega_{2}\right)$ is a Dirac function in angle variables. $\lambda$ is chosen so that the dipole linear response has a pole at $\omega=0$, corresponding to the spurious mode. This type of residual interaction has been studied extensively [8, 17, 18] and it leads to a satisfactory description of nuclear collective modes. We have used the same nonselfconsistent approach described in detail in Ref. [2] in order to estimate the magnitude of the correction $\Delta \varepsilon_{\pi l}$. For various single-particle proton levels around the Fermi level and the proton threshold, the calculated shifts $\Delta \varepsilon_{\pi l}$ are in the interval $0.1-0.3 \mathrm{MeV}$ in light $(A=16)$ and medium $(A=40-48)$ nuclei. In performing these calculations in each nucleus we have included collective modes with multipolarities up to $\approx 2 A^{1 / 3}$ and energies up to $\approx 225 \mathrm{MeV}$, see Ref. [2]. Both in multipolarities and in energy convergence was attained in our calculations. The values of $\Delta \varepsilon_{\pi l}$ thus obtained are of the same magnitude as the Nolen-Schiffer anomaly.

It is instructive to cross check these results using an independent approach. A new type 
of nuclear density functionals has been recently introduced in Refs. [5, 6], see also Ref. [9]. The main reason for seeking new functionals is to obtain a significantly more accurate reproduction of the nuclear properties (bindings energies and matter distribution of finite nuclei and infinite neutron and symmetric nuclear matter properties over a wide range of densities simultaneously) than one can achieve with the plethora of existing density functionals. A key ingredient was the introduction of the Coulomb correlation energy contribution, along the lines suggested earlier by us in Refs. [2]. However, while we have presented arguments for a significant surface contribution into the nuclear Coulomb correlation energy, Fayans [5] has chosen to parametrize the Coulomb correlation energy as a volume term:

$$
F_{\text {Coul }}^{\text {corr }}\left[\rho_{\pi}(\mathbf{r}), \rho_{\nu}(\mathbf{r})\right]=\frac{3}{4}\left(\frac{3}{\pi}\right)^{1 / 3} e^{2} h_{\text {Coul }} \int d \mathbf{r} \rho_{\pi}^{4 / 3}(\mathbf{r})\left[\frac{\rho_{\pi}(\mathbf{r})+\rho_{\nu}(\mathbf{r})}{\rho_{0}}\right]^{\sigma}
$$

Here $\rho_{0}=0.16 \mathrm{fm}^{-3}, \sigma=1 / 3$ and from a fit of the masses and radii of 100 medium and heavy spherical nuclei Fayans determined $h_{\text {Coul }}=0.941$, see also Ref. [9]. The Coulomb correlation energy determined by Fayans thus almost exactly cancels the Coulomb exchange energy, which in the Slater approximation is formally given by the same formula with $h_{\text {Coul }}=-1$ and $\sigma=0$, namely

$$
F_{C o u l}^{F o c k}\left[\rho_{\pi}(\mathbf{r}), \rho_{\nu}(\mathbf{r})\right] \approx \frac{3}{4}\left(\frac{3}{\pi}\right)^{1 / 3} e^{2} \int d \mathbf{r} \rho_{\pi}^{4 / 3}(\mathbf{r})
$$

A quick estimate of the Coulomb correlation energy contribution shows that

$$
F_{\text {Coul }}^{\text {corr }} \approx \frac{3}{4}\left(\frac{3 \rho_{0}}{2 \pi}\right)^{1 / 3} e^{2} h_{\text {Coul }} \int d \mathbf{r} \rho_{\pi}(\mathbf{r}) \approx 0.4 Z \mathrm{MeV}
$$

which leads to a typical shift $\Delta \varepsilon_{\pi l} \approx 0.4 \mathrm{MeV}$ of the same order of magnitude as estimated by us independently. An upward shift of this magnitude of the last occupied proton level in a nucleus near the proton drip line is equivalent to a shift of the proton drip line in the direction of decreasing $Z$ by a few units. A shift of this magnitude for a neutron level would be equivalent to changing the mass number by up to 5 units, see for example [19]. A similar shift due to the Coulomb interaction arises for neutron levels, but we shall not discuss this mechanism here (see however Fig. 1 and the ensuing discussion). 
In a recent preprint Brown et al. [14] show that one can generate essentially an innumerable range of phenomenological nuclear density functionals of the Skyrme type, with which the Nolen-Shiffer anomaly can be accounted for, while at the same time nuclear masses and radii can be calculated selfconsistently with very good accuracy. This lack of uniqueness for the nuclear density functional is due to the lack of an uncontroversial theoretical underpinning. It is hoped that the present results will bring the goal of a complete theoretical understanding within our grasp.

One can show that there is another related effect due to the Coulomb correlation energy, a noticeable renormalization of the proton effective mass near the Fermi surface. In the case of homogeneous nuclear matter the effective mass is defined as [16]

$$
\frac{1}{m^{*}}=\left.\frac{1}{p_{F}} \frac{d \varepsilon(p)}{d p}\right|_{p=p_{F}}
$$

where $p_{F}$ is the Fermi momentum. One can show that the effective mass renormalization $\Delta m$ can be obtained from the relation

$$
\frac{\Delta m}{m^{*}\left(m^{*}+\Delta m\right)}=\frac{e^{2}}{p_{F}} \frac{d}{d p}\left\{\int \frac{d \mathbf{q} d \omega}{(2 \pi)^{3}} \frac{\delta \chi_{0}(q, i \omega)}{\delta n(p)} \frac{1}{q^{2}\left[1-R(q, i \omega) \chi_{0}(q, i \omega)\right]^{2}}\right\}
$$

where $m^{*}$ is the proton effective mass computed in the absence of the Coulomb correlation energy and $n(p)$ is the quasiparticle occupation number probability of the state with linear momentum $p$. Using the following approximate formula (which becomes an identity at the point where the compressibility is vanishing)

$$
\left.\frac{d}{d p} \frac{\delta \chi_{0}(q, \omega)}{\delta n(p)}\right|_{p=p_{F}} \approx-\frac{4 \pi}{p_{F}^{2}} \delta\left(p_{F}-|\mathbf{p}+\mathbf{q}|\right) \delta(\omega) \mathbf{p} \cdot(\mathbf{p}+\mathbf{q})
$$

one can reduce the above expression for the effective mass shift to

$$
\frac{1}{m^{*}+\Delta m} \approx \frac{1}{m^{*}}+\frac{e^{2}}{2 \pi p_{F}} \int_{-1}^{1} \frac{x d x}{\left[1-R(q(x), 0) \chi_{0}(q(x), 0)\right]^{2}},
$$

where $q(x)=p_{F} \sqrt{2(1-x)}$. At the point where the compressibility tends to zero, the denominator $\left[1-R(q(x), 0) \chi_{0}(q(x), 0)\right]$ vanishes at $x=1(q=0)$. We have shown in Ref. [2] that the nuclear surface of finite nuclei is rather close to this regime. Since the integrand in 
Eq. (22) is positive, the integral diverges and the effective mass thus vanishes in an infinite homogeneous system. In finite nuclei this divergence is smoothed out and the pole singularity becomes a narrow surface peak [2]. The net effect is that the proton effective mass becomes smaller than the neutron effective mass, and therefore the proton level density is on average smaller than the neutron level density in nuclei. B.A. Brown argues that a somewhat similar in medium effective nucleon mass renormalization can account for the Nolen-Schiffer anomaly [9]. An earlier QCD sum-rule approach [20] substantiate such a claim. This effect, as well as the contributions arising from CSB forces [7], lead mainly to volume effects, while the many-body mechanism discussed by us leads to a surface contribution.

There is thus hope not only to generate in the near future extremely accurate nuclear density functionals, but also to be able to understand the nature of various rather subtle contributions. We anticipate as well that the study of the Nolen-Schiffer anomaly within the framework of the density functional approach will allow us to estimate the magnitude and the character (volume versus surface) of the CSB many-body effects.

\section{ACKNOWLEDGMENTS}

We thank G.F. Bertsch, E.M. Henley and G.A. Miller for discussions and a critical reading of the manuscript. This research was funded in part by DOE and by INTAS under Grant No. INTAS-OPEN-97-603. VRS would like to thank the Department of Physics of the University of Washington, for hospitality, where part of this work was done. We thank also B.A. Brown for sending us the preprint [14 prior to its publication.

[1] J.A. Nolen, Jr., J.P. Schiffer, Ann. Rev. Nucl. Sci. 19 (1964) 471; S. Shlomo, Rep. Prog. Phys. 41 (1978) 957; N. Auerbach, Phys. Rep. 98 (1983) 273. 
[2] a) A. Bulgac, V.R. Shaginyan, Nucl. Phys. A 601 (1996) 103; b) A. Bulgac, V.R. Shaginyan, JETP Letters, 62 (1995) 843.

[3] P. Hohenberg and W. Kohn, Phys. Rev. 136 (1964) B864; W. Kohn and L. J. Sham, Phys. Rev. 140 (1965) A1133; R.M. Dreizler and E.K.U. Gross, Density Functional Theory: An Approach to the Quantum Many-Body Problem, (Springer, Berlin, 1990).

[4] V.A. Khodel, V.R. Shaginyan and V.V. Khodel, Phys. Rep. 249 (1994) 1.

[5] S.A. Fayans, JETP Letters, 68 (1998) 169.

[6] S.A. Fayans, S.V. Tolokonnikov, E.L. Trykov and D. Zawischa, JETP Letters, 68 (1998) 276.

[7] G.A. Miller, M.K. Nefkens and I. Slaus, Phys. Rep. 194 (1990) 1; G.A. Miller and W.H.T. van Oers, in Symmetries and Fundamental Interactions in Nuclei, eds. W.C. Haxton and E.M. Henley (World Scientific, Singapore, 1995), p. 127; H. Müther, A. Polls and R. Machleidt, Phys. Lett. B 445 (1999) 259.

[8] P. Ring, P. Schuck, Nuclear Many Body Problem (Springer, New York, Berlin, Heidelberg, 1980).

[9] B.A. Brown, Phys. Rev. C 58 (1998) 220.

[10] In Ref. [2, a)] the lhs of the Eq. (3) should be integrated over $\omega$.

[11] J. Damgaard, C.K. Scott and E. Osnes, Nucl. Phys. A154 (1970) 12; S. Shlomo and W.G. Love, Physica Scripta, 26 (1982) 280.

[12] E.H. Auerbach, S. Kahana and J. Weneser, Phys. Rev. Lett. 23 (1969) 1253.

[13] N. Auerbach, Phys. Lett. B 36 (1971) 293.

[14] B.A. Brown, W.A. Richter and R. Lindsay, unpublished.

[15] A.B. Migdal, Theory of Finite Fermi Systems and Applications to Atomic Nuclei, (Wiley Interscience, New York, 1967). 
[16] L.D. Landau, JETP 30 (1956) 1058.

[17] A. Bohr and B.R. Mottelson, Nuclear Structure, vol. II, ch. 6 (Benjamin, New York, Amsterdam, 1974).

[18] H. Esbensen and G.F. Bertsch, Ann. Phys. 157 (1984) 255.

[19] A. Bohr and B.R. Mottelson, Nuclear Structure, vol. I, ch. 2 (Benjamin, New York, Amsterdam, 1974), Fig. 2.30 .

[20] T. Hatsuda, H. Hogaasen and M. Prakash, Phys. Rev. Lett. 66 (1991) 2851. 